Results In the first month, a total of 24 patients were seen. Eight patients were known to both teams, whilst 15 were identified as being suitable for referral to the hospice. One patient has not yet met the referral criteria. In November, half yearly results will be presented.

Conclusion This ongoing secondment is demonstrating that a significant proportion of patients under the care of the community respiratory team are suitable for referral to the hospice team. The physiotherapists are encouraging the community respiratory team to refer patients at an earlier stage, to enable patients to benefit from the range of palliative services including specialist symptom control and rehabilitative palliative care. The secondment is also proving to be a valuable way of building better relationships between the teams and a greater understanding of what each team can offer.

\section{P-218 PROVISION OF A CONSULTANT NURSE LED ACUTE HOSPITAL END-OF-LIFE CARE UNIT TO MEET UNMET NEEDS OF DYING PATIENTS}

${ }^{1}$ Dee Curless, ${ }^{2}$ Shane Moody. ${ }^{1}$ Mountbatten Isle of Wight, Isle of Wight, UK; ${ }^{2}$ Isle of Wight NHS Trust, Isle of Wight, UK

\subsection{6/spcare-2021-Hospice.233}

Introduction In October 2019 within the acute hospital on the Isle of Wight we saw an increase in patients choosing to die in hospital if they were in the last days of life. This equated to $23 \%$ of the palliative and end-of-life care teams caseload and while this was recognised we also identified a consistent number of patients dying in the emergency department, CCU and ICU that were in the last hours to last day of life and had not been offered alternative preferred places of care due to high acuity needs.

Aim This resulted in a bold case being put forward to develop a 3-6 bedded nurse- led end-of-life care unit.

Method The proposal was supported as a pilot, underpinned by working with key partners which included Mountbatten Isle of Wight. Referrals are made to the IPET team who assess; if appropriate and in agreement with medical team, patient and family, then transfer to the Wellow Unit. The Unit is managed by an experienced palliative care sister and overseen by the consultant nurses and IPET team.

Outcomes On average the Wellow Unit cares for 34 patients per month and in January 2021 at the height of the second wave of COVID-19 we cared for 64 patients. In total over the last 12 months the unit has cared for 320 patients. After a successful 12 months of being in place and increasing beds to meet COVID-19 demand in 2020-21, the unit has now been made permanent.

Conclusion This development rightfully challenged this partnership working which ultimately strengthened the position we now find ourselves in. In sharing knowledge, skills and workforce resources we are now in a position to consistently meet the needs of the dying patient in the acute hospital.

\section{P-219 KEEPING AN EYE: NURSE- LED TELEPHONE SURVEILLANCE IN THE HOSPICE SETTING}

Kate Dreyer, Kate Marley, Carolyn Julie Bellieu, s Emma Nelson, Louise Cook, Jenny Warren, Carolyn Hamilton, Clare Doyle, Dawn Porter. Liverpool University Hospitals NHS Foundation Trust, Liverpool, UK

10.1136/spcare-2021-Hospice.234
Prior to the start of the COVID-19 pandemic, Woodlands Hospice Wellbeing and Support Centre ran several regular groups plus nurse-led clinics for patients well enough to attend the hospice. When the pandemic started it was clear that this would no longer be an option as most of the patients were in the shielding category.

Clearly the needs of these patients would not go away but the community palliative care and district nursing services could not be expected to take on the role that the hospice had previously played, as they also faced increased demand due to the pandemic.

Out of necessity and working towards a new city-wide integrated palliative care model, a decision was made to move to a system of triage coordinators and regular nurse led telephone clinics to monitor these patients. The nursing team underwent an education programme to enhance their triage and symptom management skills and they had ready access to the medical team for support if there were problems.

Consultations are carried out by telephone or video consultation as well as face-to-face where needed. The nursing team gradually took over the surveillance role of the medical clinics to free capacity for urgent medical appointments so patients could be assessed by the doctors when problems arise or when symptoms are more complex. The triage coordinator now looks at all referrals coming into the hospice and assesses the patient's needs to ensure the patient is linked into the most appropriate services. All this integrated working has reduced the time from referral to comprehensive specialist nursing assessment in the hospice from two weeks to within five working days. The working relationships with all teams has become closer and more effective, and patients are still able to access services despite the restrictions that the pandemic has brought.

\section{P-220 NEVER A DULL MOMENT!}

Dee Curless. Mountbatten Isle of Wight, Isle of Wight, UK

\subsection{6/spcare-2021-Hospice.235}

Introduction As Mountbatten grew and expanded its locality, I was asked to consider working with hospital colleagues across two hospitals- one a District General Hospital and the other a University Trust - to build on existing relationships whilst forging new ones. This was a daunting prospect, considering myself a community nurse for the last 24 years! I also returned as a Clinical Teaching Fellow to University, delivering palliative and end-of-life care education to pre- and postregistration students.

Aim To build and grow new and existing relationships, sharing and developing nursing practice.

Method Working clinically with acute teams to augment patient care and deliver Enhanced Supportive Care clinics in a University Trust in parallel with a Medical Consultant to effectively double the clinic capacity. Also, delivering education and training around palliative care initiatives to both staff and students.

Outcomes The diversity of environments has taken me from practice in the community working with patients and families one day, such as supporting a couple to renew their wedding vows after many years together, knowing that time is short, to delivering care in an acute setting such as ICU the next day where a patient and family need support in symptom 
management and psychological care to enable weaning from ventilation and a comfortable death.

Conclusion This new and unique role has reinforced the value of Consultant nursing practice at a clinical level, influencing and shaping nursing care in a live way. The transferable skills held have enabled dynamic nursing that could be seen as a blueprint for promoting a workforce development that is more resilient for the future, valuing the differing perspectives of nursing practice in both primary and secondary care.

\section{P-221 A TALE OF TWO HOSPICES: THE DEVELOPMENT OF A UNIQUE SERVICE MODEL OF HOSPICE CONSULTANT SUPPORT}

${ }^{1}$ Eilidh Dear, ${ }^{1}$ Jill McKane, ${ }^{2}$ Amy Proffitt, ${ }^{1}$ Paul Coulter, ${ }^{1}$ Linda McEnhill, ${ }^{2}$ Robert George. ${ }^{1}$ Ardgowan Hospice, Greenock, UK; ${ }^{2}$ St Christopher's Hospice, London, UK

10.1136/spcare-2021-Hospice.236

Background The Association of Palliative Medicine of Great Britain and Ireland (APM) state 'Palliative medicine is at a turning point in terms of delivering new service models' (Workforce Committee of the APM, 2019).

Ardgowan Hospice has had consultant recruitment challenges. Explanations include geography and no Clyde palliative trainee placements. There was an estimated 56 vacant UK consultant posts in 2018. Too few trainees and the removal of the CESR process means the number of prospective consultants is insufficient for service demand. A changing teaching curriculum and seven-day working creates concerns for junior-tier rotas which will impact the consultant on-call. With an ever-increasing demand of services, innovative staffing solutions are required so palliative medicine can continue to flourish in hospitals, hospices, and community.

Ardgowan Hospice (AH) and St Christopher's Hospice $(\mathrm{SCH})$ developed an innovative service model for hospice consultant support.

Aim To develop and evaluate a remote consultant service model.

Method Quality improvement methodology enabled the design, development, and evaluation of remote consultant framework. Plan Do Study Act (PDSA) cycles, cause and effect analysis, field analysis, survey, and audit were all utilised.

Results

- In 2019, PDSA cycles showed that it was imperative that shared policies, guidelines, and protocols were established to provide seamless patient care.

- Quality improvement analysis highlighted important themes for cohesive working. These were team-work, flexibility and adaptability, continuity of care, as well as high quality handovers.

- Innovative IT solutions were developed to provide efficient communication within clinical governance structures.

- A 2021 audit showed 11 communications with SCH about 12 patients over a three-months period. This facilitated appropriate senior advice when required.

- A 2021 survey was conducted. The average satisfaction score was $8.5 / 10$. A number of qualitative themes were identified.

Conclusion $\mathrm{AH}$ and $\mathrm{SCH}$ have shown that by completing a quality improvement project that it is possible to create an innovative remote consultant service provision model.

\section{P-222 DESIGNING A MOBILE APP WHICH SUPPORTS UNPAID CARERS TO ADMINISTER SUBCUTANEOUS INJECTIONS}

${ }^{1}$ Claudia Hopkins, ${ }^{2}$ Imogen Eastwood, ${ }^{3}$ Marlise Poolman, ${ }^{1}$ Ivor Williams, 'Lily Hoskin, ${ }^{1}$ Cat Kilkenny, ${ }^{1}$ Jonathan Gregory. ${ }^{1}$ Helix Centre, Imperial College, London, UK; ${ }^{2}$ Central North West London NHS Trust, London, UK; ${ }^{3}$ Bangor University, Bangor, UK

\subsection{6/spcare-2021-Hospice.237}

In the UK, most people wish to be at home when they die. Unpaid carers (family and friends) play a key role in ensuring a home death and many are willing to be trained to administer as-needed subcutaneous injections for breakthrough symptoms, to ensure timely symptom control for the person they are caring for. A team in North Wales developed the CARiAD package to train and support such carers, a new practice to most of the UK.

It is hypothesised that a digital app may be beneficial for both carers and the healthcare teams. It could provide more guidance, improve data collection, help facilitate monitoring through the real-time collection of data and support audit data collection. Therefore the Helix Centre, Central North West London (CNWL) NHS Trust and the CARiAD team have collaborated to design and develop a mobile app. We acknowledge that a digital product will not be right for every carer but it is essential to understand whether a digital offering can deliver the intended benefits.

A user-centred design approach was adopted by conducting moderated usability sessions with carers who had previously given subcutaneous as-needed medications. Testing user flows, content and barriers of using paper and digital versions together. After each round, we synthesised the qualitative feedback and made improvements. A pre-pilot study of the app is commencing imminently which will inform a formal pilot study in CNWL of a digital-first offering. If successful a healthcare practitioner view will be developed to aid remote monitoring and support carers.

The design process worked as intended. Unforeseen usability and accessibility issues were identified. Iterative improvement of the app has delivered a product that is well aligned with carers' perspectives and knowledge. We hope this will ensure that the app is easier and safer to use when supporting carers to administer and record as-needed subcutaneous injections.

\section{P-223 SOCIAL WORK LED CARE COORDINATION WITHIN A COMMUNITY PALLIATIVE CARE TEAM}

Catherine Thompson. Kirkwood Hospice, Huddersfield, UK

10.1136/spcare-2021-Hospice.238

The Kirkwood recognised through its user engagement with patients and carers that coordinating palliative health and social care for patients and carers can be extremely complex, confusing and stressful. It appeared to be a contributing factor to carer stress. The crossover from social care to healthcare was often very abrupt with no lead support along the way. It was identified that having a lead service able to remove some of the strain and pressure was needed with the right level of skills and knowledge to support and navigate the system. 